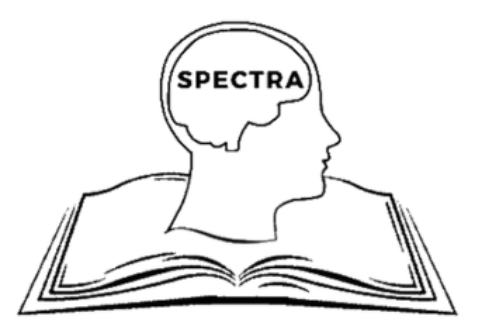

Spectra Undergraduate Research Journal

Volume 1, Issue 2

Office of Undergraduate Research

University of Nevada, Las Vegas

digitalscholarship.unlv.edu/spectra/

\begin{tabular}{|c|}
\hline Category \\
\hline Health \& Natural Sciences \& Engineering $>$ Health Sciences $>$ Public Health \\
\hline Received \\
\hline March 2, 2021 \\
\hline Accepted \\
\hline July 19,2021 \\
\hline Published \\
\hline August 13, 2021 \\
\hline Article Title \\
\hline $\begin{array}{l}\text { Qualitative Review of Educational Interventions for Improving Physical Activity Behaviors in Older } \\
\text { Asian Americans in the United States }\end{array}$ \\
\hline Authors \\
\hline Raisa Kabir $(\mathrm{RK})^{1}$, Manoj Sharma $(\mathrm{MS})^{2}$, Sayeda Tazim Zaidi $(\mathrm{STZ})^{3}$, and Chia-Liang Dai (CLD) ${ }^{4}$ \\
\hline Author Affiliations \\
\hline 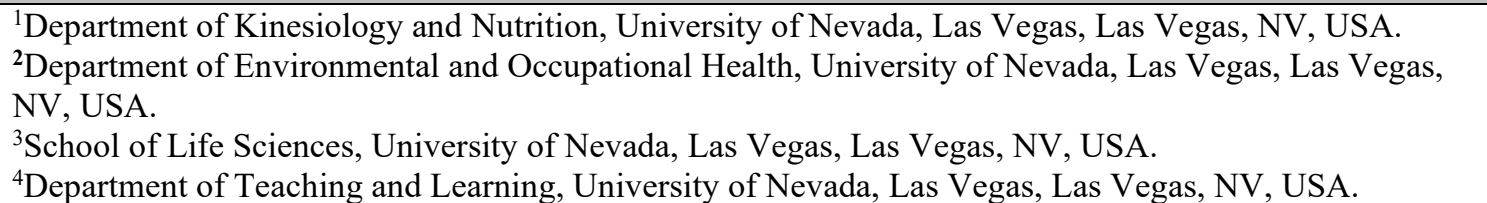 \\
\hline Corresponding Author \\
\hline *Raisa Kabir, kabir@unlv.nevada.edu \\
\hline Author Contributions \\
\hline $\begin{array}{l}\text { RK: Contributed conceptualization, source curation, data collection, drafting of paper, formal analysis, } \\
\text { methodology, writing of the original draft, and reviewing and editing of the manuscript. } \\
\text { MS: Contributed conceptualization, formal analysis, and reviewing of the manuscript. } \\
\text { STZ: Drafting of paper and reviewing and editing of the manuscript. } \\
\text { CLD: Contributed conceptualization, formal analysis, and reviewing of the manuscript. }\end{array}$ \\
\hline Copyright \\
\hline $\begin{array}{l}\text { (c) Articles in Spectra are freely available under a Creative Commons Attribution License } \\
\text { (CC BY 4.0) which allows others to re-use the work without permission as long as the } \\
\text { work is properly cited. }\end{array}$ \\
\hline ISSN \\
\hline $2766-7227$ \\
\hline $\begin{array}{r}\text { Data Availability Statement } \\
\end{array}$ \\
\hline $\begin{array}{l}\text { The study did not collect any primary data. All secondary articles are available through the UNLV } \\
\text { University Libraries. }\end{array}$ \\
\hline Conflicts of Interest \\
\hline The authors declare that no conflicts of interest exist. \\
\hline Ethical Considerations \\
\hline $\begin{array}{l}\text { Given that this research paper did not involve primary human or animal subjects, no IRB/ IACUC or other } \\
\text { ethical approval was needed. }\end{array}$ \\
\hline Funding \\
\hline No funding was used to conduct this research. \\
\hline Recommended Citation \\
\hline $\begin{array}{l}\text { Kabir, R., Sharma, M., Zaidi, S. T., \& Dai, C.. (2021). Qualitative review of educational interventions for } \\
\text { improving physical activity behaviors in older Asian Americans in the United States. Spectra } \\
\text { Undergraduate Research Journal, } 1(2), 56-67 . \text { https://doi.org/10.9741/2766-7227.1010 }\end{array}$ \\
\hline
\end{tabular}




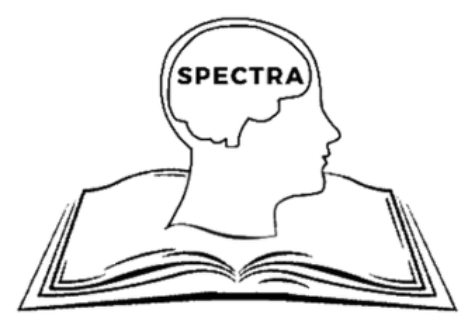

Spectra Undergraduate Research Journal

Volume 1, Issue 2

Office of Undergraduate Research

University of Nevada, Las Vegas

digitalscholarship.unlv.edu/spectra/

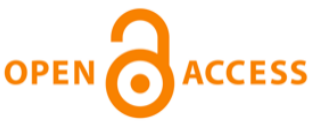

\section{Comprehensive Review of Educational Interventions for Improving Physical Activity Behaviors in Older Asian Americans in the United States}

Raisa Kabir ${ }^{1}$, Manoj Sharma ${ }^{2}$, Sayeda Tazim Zaidi ${ }^{3}$, and Chia-Liang Dai ${ }^{4}$

\author{
Author Affiliations: \\ ${ }^{1}$ Department of Kinesiology and Nutrition, University of Nevada, Las Vegas, Las Vegas, NV, USA. \\ ${ }^{2}$ Department of Environmental and Occupational Health, University of Nevada, Las Vegas, Las Vegas, NV, USA. \\ ${ }^{3}$ School of Life Sciences, University of Nevada, Las Vegas, Las Vegas, NV, USA. \\ ${ }^{4}$ Department of Teaching and Learning, University of Nevada, Las Vegas, Las Vegas, NV, USA.
}

\begin{abstract}
Physical activity (PA) provides numerous health benefits and minimizes chronic diseases and mental health issues, especially in older adults. The national survey data indicated that only $27.3 \%$ to $44.3 \%$ of older adults meet the recommended PA guidelines of 150 to 300 minutes/ week. There is limited data on PA behavior among older Asian American (AA) adults as very few to no local/national surveys have targeted these marginalized subgroups. This comprehensive review aims to identify existing PA intervention strategies and to update current knowledge on the effectiveness of these interventions to improve PA behavior among aging AA in the US. The study included publications from the last five years (2016 to 2021) on all PA interventions with older AA adults. Data were abstracted for study designs, associated conditions, subgroups focused, theoretical frameworks, sample sizes, intervention strategies, and PA outcomes. A total of nine intervention studies met the inclusion criteria based on age, ethnicity and racial group, year of publication, United States-based, and Englishonly publication. Four studies used randomized control trial designs, five used pretest-posttest designs, one used a single-group time series design, and one was a qualitative study. The studies included in this paper, aside from the qualitative research, did not use behavioral theories and utilized small sample sizes. It is crucial to develop long-term effective interventions which will help to increase PA and decrease sedentary behaviors in older AA. To achieve these goals, more research addressing and utilizing theory-based PA promotion intervention with larger sample sizes.
\end{abstract}

Keywords: physical activity, intervention, aging Asian American, comprehensive review

Physical activity (PA) is the movement of the human body using energy (Caspersen et al., 1985). PA is a crucial component of maintaining a healthy lifestyle. It reduces the risks of chronic diseases and mental illness such as obesity, cardiovascular disease, and certain types of cancer (Deng et al., 2019). According to the U.S. Physical Activity Guidelines for Americans (PAGA), adults should participate in the moderate aerobic activity of 150 to 300 minutes/week or vigorous aerobic activity of 75 to 150 minutes/week. Additionally, it's suggested that they participate in muscle-strengthening resistance exercises at least two or more times/week to activate all major muscle groups. (U.S. Department of Health and Human Services, 2018). However, the U.S. Department of Health and Human Services also suggested these 
guidelines do not apply to adults over the age of 65 with one or more chronic illnesses. Instead, it is recommended for them to stay physically active as much as they can depending on their health conditions and body strength. The national survey data indicated that only $27.3 \%$ to $44.3 \%$ of older adults meet the recommended PAGA guidelines of 150 minutes/ week (Keadle et al., 2016). Asian Americans (AA) are one of the rapidly growing ethnic/racial groups in the United States (Chesla et al., 2016). However, there is scant research on the PA and healthy lifestyle in aging AA adults; even less research is available for minority subgroups of AA.

This comprehensive review aims to identify existing PA intervention strategies and to update current knowledge on the effectiveness of these interventions to improve PA behavior among aging AA in the US. The Population, Intervention, Comparison, and Outcome framework (PICO) was used to determine inclusion/exclusion factors (Richardson et al., 1995). The Preferred Reporting Items for Systematic reviews and Meta-Analysis (PRISMA) were utilized to extract and analyze the collected data (Page et al., 2021). The interventions identified in this study were implemented to different populations and subgroups including Chinese, Korean, Filipino, and Indian Americans. The intervention designs included multilingual programs, group style, and pretest-posttest which allowed participants from different subgroups of AA to receive intervention in their native languages, work in groups, and report before and after intervention results. The results of this study demonstrated the effectiveness and retention factors of home-based health promotion prevention programs, languagetranslated prevention programs, lifestyle intervention, and diet-based intervention programs.

This study is significant because there is limited recent literature on PA behavior and the effectiveness of PA intervention among AA even though this minority group has high rates of diseases such as arthritis, obesity, blood pressure, and other cardiovascular diseases. The findings from this study can be used to identify the effectiveness of PA interventions targeted to specific subgroups of AA which would allow researchers to design effective interventions in the future to improve PA among AA.

\section{Methodology}

Literature Search and Inclusion Criteria: An extensive primary literature search was conducted in MEDLINE/ PubMed Central, CINAHL, and EBSCO databases using the key terms: physical activity and Asian Americans. The PICO framework helped structure the literature question and develop search strategies (Richardson et al., 1995). The PICO framework was used to determine and design possible inclusion and exclusion criteria for the literature review on Microsoft Excel (Table 1). The inclusion criteria were: (1) articles published in the past 5 years (20162021), (2) peer-reviewed articles, (3) articles published in the English language, (4) articles published in the United States, and (5) articles published on Asian Americans of 55+ years and older. Both qualitative and quantitative research studies were included. The search was conducted periodically through mid-January to March 2021 using customized key terms in selected search engines. The searching strategies used in MEDLINE and PubMed: TOPIC (physical activity interventions) and TOPIC (Asian American Adults) during the timespan from 2016 to 2021. In CINAHL and EBSCO databases, a combination of PA interventions and AA adults was searched, and limited full-text publications were located from 2016 to 2021 . A total of 27 literature results were located.

Exclusion Criteria: Grey literature, non-peerreviewed articles, and philosophical and opinion-based papers were excluded during the literature retrieval phase. Additionally, studies conducted outside of the United States, not meeting the age group, duplicates, and full text not being available were excluded from this study.

Data Extraction and Synthesis: The data extraction process was performed using Preferred Reporting Items for Systematic reviews and Meta-Analyses (PRISMA) guidelines (Figure 1). The new PRISMA guidelines (2020) allowed us to accurately report eligible studies that meet the inclusion guidelines, why they fit the criteria, and how they can be incorporated into the study (Page et al., 2021). Eligibility screening was conducted independently by three researchers. Articles were retrieved based on the title. If the titles had key terms: physical activity and Asian American (or subgroups of AA), then the abstracts were reviewed. During the abstract screening stage, if PA, 
AA, and the United States were mentioned, then further screening was performed on the full text of the articles. If terms such as physical inactivity, involvement in sports, sedentary behavior, obesity, healthy lifestyle, and exercise were located within the titles, then detailed assessments were conducted on the abstracts. Figure 1 utilized three major databases to identify research articles. The excluded studies included $(n=9)$ studies that were repeated in MEDLINE/ PubMed Central, CINAHL, and EBSCO databases. An addition of $(n=490)$ articles that were ineligible due to automation tools, and $(n=5)$ articles were excluded due to other reasons. After screening based on title and abstract, 13 articles were included. Next, the full-text articles were assessed, and 11 studies met the inclusion criteria. Four studies used randomized control trials (RCTs), five studies used pretest-posttest designs, one study used single-group time-series design, and one qualitative study design.

Data synthesis was conducted separately for each physical activity article which included articles on the effectiveness of the intervention and general recommendations. Two investigators independently summarized the articles based on author, year of publication, type and description of the intervention, target population, setting, study design, sample size, and outcomes (effectiveness, post-intervention, and retention rate). The results of the studies were summarized and identified both positive and negative outcomes of the interventions.

\section{Results}

The included studies were diverse in terms of intervention strategies that were incorporated. The studies used a variety of intervention strategies such as language-based, web-based, educational-based, theory-based, community-based target intervention, and practice-based targeted intervention. The studies were categorized based on intervention type, study type, target population, sample size, and outcome (Table 2). The studies targeted three major ethnicities of older AA - Chinese, Filipinos, and Koreans.

Practice-Based Intervention (n=1): An et al. (2018) incorporated a pilot education stroke prevention program for older Korean Americans. The study examined the practicability and the effect of the stroke prevention program by measuring knowledge about stroke, dietary behaviors, and PA behaviors. The sample size included 73 Korean Americans with an intervention group consisting of 37 individuals and 36 participants in the control group. After the completion of the invention, the intervention group demonstrated improvement in the intake of sodium $(\mathrm{p}=0.048)$ and overall knowledge in stroke $(\mathrm{p}=0.001)$ compared to the control. PA assessed by the International Physical Activity Questionnaire-Short Form (IPAQ-SF) showed marginal effects in the intervention group. While the differences between groups were not significant, the post-test assessment indicated a mean of 7,900 steps per day in the experimental group. The study concluded the overall effectiveness and feasibility of stroke knowledge and PA behavior in older Korean Americans.

Combined Language and Practice-Based Tailored Intervention ( $\boldsymbol{n}=2)$ : Chesla et al. (2016) utilized a language-translated program (Group Lifestyle Balance Program) for diabetes prevention programs for Chinese Americans $(\mathrm{n}=25)$. The program incorporated 16 sessions administered over six months in separate English and Mandarin language-based groups. This program adapted the CABLE intervention program which included 12 months in three phases: core phase, the transition phase, and supports phase. Self-reported questionnaires included in the study suggested there were improved dietary intake and overall increased PA. The results included self-reported PA and weekly METS increased $(\mathrm{p}<0.01)$. The participants indicated to have a $5.4 \%$ loss of body weight through six months. The overall study showed high satisfaction and acceptance of the program.

Deng et al. (2019) conducted a pilot study for Chinese American cancer survivors $(\mathrm{n}=50)$ through a community-based organization for a healthy lifestyle. The study utilized The Reach out to ENhanceE Wellness (RENEW) program with information on PA and dietary information in Mandarin Chinese. The participants' health profiles were measured using a self-report-36 (SF-36) questionnaire. Based on the post-intervention, $96 \%$ of the participants participated in a daily 30-minute walk whereas the initial baseline was at $86 \%$. The study indicated that compared to the baseline, there is a higher frequency of PA and consumption of vegetables at the post-intervention survey. Additionally, 58\% of the participants fell 
within the healthy weight range after receiving the intervention.

\section{Combined Language and Web-based Tailored} Intervention ( $\boldsymbol{n}=\mathbf{1})$ : Chee et al. (2016) incorporated practical issues to create Web-based PA promotional programs for Chinese and Korean American midlife women. The study was divided into two phases. Phase 1: feasibility test and expert review and phase 2: a preliminary RCT. Phase 1 incorporated a one-month web-based survey forum. The results directed the refinement of the overall intervention. Phase 2 utilized an RCT (initial pretest, post one month, and post three months) consisting of $(n=69)$ Chinese or Korean American midlife women. The results were categorized into four important components of the intervention: interpreters' language accuracy, response rate, cultural sensitivity, retention of content, interest, and logistical issues.

\section{Combined Practice and Web-based Intervention} $(\boldsymbol{n = 5})$ : Bender et al. (2017) implemented a mobile health weight loss program to reduce risks of chronic disease, specifically type 2 diabetes in Filipino Americans $(n=45)$. The study utilized a randomized control trial and was divided into phase 1 with an active waitlist control and phase 2 with a three-month sequel. The PilAm Go4Health intervention used an accelerometer, a cell phone application that records food/calories, weight, and steps, and virtual support through social media. The findings from the study concluded that study completion and participant retention were at $100 \%$ and almost perfect attendance at all the seven intervention office visits.

Bender et al. (2018) implemented a Fit and Trim intervention study for the Diabetes Prevention Program to reduce weight by $5 \%$ through PA and healthy eating. The study utilized a randomized control trial for three months using mobile technology (phase 1) with three-month maintenance follows up (phase 2). The findings of the study concluded a $91 \%$ participant retention rate. The experimental group had a higher weight in comparison to the comparison group. After the administration of the intervention, there was a significant weight reduction reported. The study also indicated that $41 \%$ of participants attained a $5 \%$ weight loss.

Chee et al. (2019) incorporated an internetbased PA program for Korean and Chinese American middle-aged women. The program was broken into phase 1 for feasibility test and expert review and phase 2 for a pilot RCT. The program utilized three webbased components: message boards, educational sessions in Korean, English, and Chinese (Mandarin), 1-1 coaching sessions, and available resources. Four bilingual researchers translated the educational modules to Chinese and Korean. The study had cultural sensitivity issues, low-interest levels, poor response rates, and reduced retention rates. A significant increase in PA (i.e., active living habits) was observed in both groups at the three-month post-intervention assessment ( $\mathrm{p}=0.0353)$.

Chee et al. (2020) utilized an online program to promote PA to reduce cardiovascular symptoms. The study was divided into three portions - baseline, after 1-month posttest, and three-month posttest. The intervention group showed a significantly higher PA involvement. The control group showed a higher PA score $(\mathrm{p}=0.04)$ than at the baseline test. The intervention group indicated higher total PA scores $(\mathrm{p}=0.07)$ than at the baseline test. The overall results supported the preliminary findings for PA but did not fully support cardiovascular symptoms.

Im et al. (2017) utilized a practice and webbased intervention incorporating educational modules to promote PA to improve symptoms for menopause in AA midlife women. The study incorporated a randomized pretest-posttest measure with three assessment time points: pretest, 1-month posttest, and 3-month posttest and utilized Kaiser Physical Activity Survey (KPAS) and midlife women's symptom index (MSI). The control group experienced decreased total severity scores. Both the control $(\mathrm{p}<0.10)$ and intervention group $(\mathrm{p}<0.001)$ improved in total severity scores which improved their overall active living habits over time. The results of the program indicated potential preliminary efficacy in increasing overall PA.

Language-Based Intervention ( $\boldsymbol{n}=\mathbf{1})$ : Jih et al. (2016) evaluated the effectiveness of an in-language program utilizing two lectures of print materials on PA and nutrition guidelines. The study included 756 Chinese Americans aged 50 to 75 years and assigned them randomly into two groups - printed interventions $(\mathrm{n}=357)$ and lectures plus printed intervention $(\mathrm{n}=361)$. The intervention incorporated knowledge on vegetable 
and fruit intake and PA levels with intervals from pre to six months post-intervention. The study reported that at baseline, the knowledge and adherence to recommendations are low. Printed materials increased vegetable intake and PA adherence to fruit intake and PA recommendations. The lecture with printed materials led to an increase in all six outcomes. Both the printed intervention and lecture with printed intervention increased nutrition and PA behaviors.

Theory-Based Tailored Intervention ( $n=1)$ : Chang et al. (2018) utilized the Health Belief Model (HBM) consisting of four HBM constructs to understand the behavior of walking as an exercise for stroke. The study included Chinese and Korean Americans aged 60 years or older with a previous history of high blood pressure and stroke. Stroke prevention incorporated lifestyle changes with diet and PA. The study evaluated perceived stroke susceptibility, perceived stroke severity, perceived benefits, and perceived barriers and provided possible prevention measures. The focus group discussions (FGDs) revealed that stroke might be caused due to overeating, extreme temperature changes, and vigorous exercise. However, walking is crucial for overall health.

\section{Discussion}

This comprehensive review included inclusion criteria of publication from the last 5 years (20162021). This is because having a more recent time search allowed us to include more relevant and current research studies on the topic and capture the essence of present literature and trends. The initial literature search included both age ranges of, $55+$ and $65+$. However, the inclusion criteria included aging AA adults of the age of $55+$ because there was a larger number of studies directed at aging AA adults. Additionally, aging AA adults starting at the age of 55+ were included because they are likely to show first symptoms of body aging and decrease in overall performance skills (Puciato et al., 2017). These symptoms are detrimental to an individual's performance in society and their overall health.

The study aimed to report on the efficacy of culturally adapted PA interventions among older AA in the US based on a comprehensive review of the existing literature. This study yielded several important key points on PA intervention among older AA adults.
The general low rates of PA among the different ethnic/racial subgroups of AA highlights the need for more programming for this subgroup. It also underscores the importance of considering sociodemographic factors such as location and neighborhood, language, citizenship status, and access to health care when designing successful interventions. Although chronic health issues often serve as possible barriers against PA, older AA adults are often encouraged by health professionals to be more physically active (Bender et al., 2017). AA is one of the fastest-growing racial/ ethnic groups in the US, yet there is a lack of research literature regarding interventions to promote PA-related behaviors (Chesla et al., 2016). There is a need to have more research on PA to promote interventions for older AA adults.

Although many of the included studies do not specifically test PA improvement, it does, however, mention PA as an outcome measure while testing changes in disease symptoms. The results of the included studies indicated that the interventions improved PA behavior among older AA who were initially less physically active (An et al., 2018; Chesla et al., 2016). These findings are encouraging and point to the fact that PA is a modifiable behavior even among the aging population of AA. The study conducted by Jih et al. (2016) included a large sample size with very high retention rates. Three studies reported retention rates ranging from $82 \%$ to $100 \%$ (Bender et al., 2017, Bender et al., 2018, \& Jih et al., 2016). These findings are also significant and point to the interest among older AA to participate in such programming if it is available. Planners and policymakers should take note of this and plan more PA programs for older AA adults.

The studies included in this review utilized different methodologies to design various PA interventions. The research designs among these identified interventions included four randomized control trial studies (An et al., 2018; Bender et al., 2017; Bender et al., 2018; \& Jih et al., 2016), five pretest-posttest design studies (Chee et al., 2016; Chee et al., 2019; Chee et al., 2020; Deng et al., 2019; \& Im et al., 2017), one single group time-series design study (Chesla et al., 2016), and one qualitative study design (Chang et al., 2018). This finding of few RCTs underscores the need to have more RCTs which are the gold standard for evidence in science. The duration and 
types of PA interventions also varied. There is a need to have more standardization of the duration and types of PA interventions.

Due to the high ethnic/racial diversity among AA, it is important to consider possible cultural/racial barriers when designing PA interventions (Chesla et al., 2016). AA cannot be considered a monolithic entity as there are substantial differences with subcultures of AA. There is a need to understand and incorporate the unique cultural nuances of each Asian subgroup in designing PA promotion interventions. For example, the intervention strategy used by An et al., (2018) was effective because the researchers addressed the unmet needs of Korean Americans by educating them on stroke prevention and healthy diets based on traditional dishes. The knowledge on healthy lifestyle and diet plans may differ for other sub-Asian populations. Additionally, precautions must be taken when choosing intervention strategies so that they are culturally relevant. For example, square dancing and tai-chi may be more appealing to Chinese Americans while yoga may be more appealing to Indian Americans.

The target population's education level should also be considered as it may become an essential factor in implementing a successful PA intervention strategy. For example, the mobile-phone-based intervention strategy used by Bender et al., (2017) was remarkably successful with a $100 \%$ retention rate because the targeted Filipino American adults were highly educated and quickly adapted to mobile technology for tracking health behavior. On the other hand, Chee et al. (2016) indicated that web-based PA interventions for Chinese and Korean American women made it challenging to recruit and retain participants and $30.43 \%$ dropped out by three months post-intervention. The newer modalities that utilize technology are quite appealing to the researchers and planners, but they must consider the fact that older community members may not be receptive or well prepared to receive these new technologies. Hence, investing in acclimatizing and slowly training older AA in the use of technology must be undertaken.

Only one study utilized a behavioral theory explicitly and that was HBM (Chang et al., 2018). The field of health promotion has grown from knowledge, attitudes, practice (KAP)-based interventions to skill- based interventions to earlier theories like HBM to now multi theory models (Sharma, 2017). There is a need to utilize contemporary behavioral theories and models that can increase the chances of greater behavior change concerning PA behavior among older AA through brief and precise interventions.

Importance to the Field: There is limited data available on PA behavior among older AA adults. This study is significant to public health and health sciences because it provides a comprehensive review of existing literature from the last five years on PA interventions among older Chinese, Filipino, and Korean American adults. Similar interventions with higher retention rates can be adapted for other ethnic/racial groups.

Implications for Future Research and Future Direction: There is a need to design PA intervention strategies targeted toward and tailored for specific ethnic/racial subgroups within the AA population. Future studies should utilize larger sample sizes, randomized control trials, and behavioral theories to test the efficiency and efficacy of PA interventions. Additionally, various combinations of targeted and tailored PA interventions can be tested for costeffectiveness. A better understanding of the cultural, language, practice, theory, and web-based intervention will potentially increase new initiatives and policies for the marginalized AA communities.

This study will contribute to better personal health, promote preventative care, and create an intervention curriculum for physical inactivity. Some of the future directions for this particular study may include a deeper analysis of the intervention types targeted for marginalized AA communities. Furthermore, it may also be beneficial to understand how cultural perception of PA behavior affects the type of intervention based on the different community settings. Some future studies should be dedicated to utilizing a community-engaged approach to understand social and behavioral differences of AA sub-groups and design efficient health policies.

Limitations of the Included Interventions: Although a majority of the interventions reported positive impacts on participants' PA behaviors at the post-intervention assessments, only four interventions applied the randomized controlled trial designs, and only one intervention incorporated a theory. Also, a wide range of PA measures was used across included interventions 
and was limited to self-reported questionnaires. Additionally, several interventions included in this study used small sample sizes and did not include all ethnic subgroups of AA. These findings suggested the need to standardize outcome measures and utilize theory-based and rigorous research designs. Finally, publication bias may have played a role in interpreting the results as the studies with positive outcomes may have higher rates to be published.

Limitation of the Review: There were a few limitations in this review. There are very few studies on this PA published within the last five years (2016-202). This review focused on finding articles from four major databases: MEDLINE/ PubMed Central, CINAHL, and EBSCO databases. Gray literature was excluded from the compilation of the articles used in this study. The studies within this review only included articles published in the English language and the United States-based studies; articles based outside of the United States and other languages were excluded.

\section{Conclusions}

The major findings indicated that most of the studies incorporated a mixture of culturally adapted programs with different phases to ensure monthly progress (Bender et al., 2017; Bender et al., 2018; Chee et al., 2016; Chesla et al., 2016). The different interventions showed positive health benefits such as weight loss, stabilized type 2 diabetes status, regular estrogen levels, improved menopausal symptoms, improved sleep-related symptoms, and reduced cardiovascular-metabolic risks such as hypertension, stroke, and hyperlipidemia (An et al., 2018; Bender et al., 2017; Bender et al., 2018; Chee et al., 2020; Chesla et al., 2016; Deng et al., 2019; Jih et al., 2016; Im et al., 2017). According to Jih et al. (2016), the educational intervention has also influenced an increase in healthy eating with more fruit and vegetable consumption.

This review can contribute to developing future health research studies to increase overall PA and decrease sedentary behavior among the older AA community from different socio-demographic backgrounds. Additionally, it can be used as a foundation to design an intervention to promote PA among the Asian American population. This study utilized detailed analysis to examine 11 peer-reviewed articles to assess the effectiveness of existing PA intervention designs. The intervention designs included language-based interventions, practice-based interventions, combined language, and practice-based tailored intervention, combined language and webbased tailored intervention, combined practice, and web-based intervention, and theory-based tailored intervention. The articles included in this study indicated potential benefits as well as limitations of utilizing various PA interventions. This study provides insights into peer-reviewed literature concerning the effectiveness of intervention for PA in older AA adults. The results of the studies indicated that interventions incorporating multilingual translators adequate in various Asian languages would encourage participant engagement and maintain a high retention rate (Chee et al., 2016). Additionally, different AA cultural heritage needs to be taken into consideration when creating intervention programs (Chee et. al, 2020).

\section{Acknowledgments}

I would like to thank my faculty mentors Dr. Sharma and Dr. Dai for their patience and guidance throughout this project. We want to acknowledge Sayeda Tazim Zaidi for her contribution to editing this project.

\section{References}

An, M., Nahm, E. S., Shaughnessy, M., Storr, C. L., Han, H. R., \& Lee, J. (2018). A pilot primary stroke prevention program for elderly Korean Americans. The Journal of Neuroscience Nursing, 50(6), 327-333. https://doi.org/10.1097/JNN.00000000000003 97

Bender, M. S., Cooper, B. A., Park, L. G., Padash, S., \& Arai, S. (2017). A feasible and efficacious mobile-phone based lifestyle intervention for Filipino Americans with type 2 diabetes:

Randomized controlled trial. JMIR Diabetes, 2(2), e30. https://doi.org/10.2196/diabetes.8156

Bender, M. S., Cooper, B. A., Flowers, E., Ma, R., \& Arai, S. (2018). Filipinos Fit and Trim - A feasible and efficacious DPP-based intervention trial. Contemporary Clinical Trials Communications, 12, 76-84. https://doi.org/10.1016/j.conctc.2018.09.004 
Caspersen, C J, Powell, K E, \& Christenson, G M. (1985). Physical Activity, Exercise, and Physical Fitness: Definitions and Distinctions for Health-Related Research. Public Health Reports (1974), 100(2), 126-131.

Chang, E., Choi, S., Kwon, I., Araiza, D., Moore, M., Trejo, L., \& Sarkisian, C. (2018). Characterizing Beliefs about Stroke and Walking for Exercise among Seniors from Four Racial/Ethnic Minority Communities. Journal of Cross-Cultural Gerontology, 33(4), 387-410. https://doi.org/10.1007/s10823-0189356-6

Chee, W., Kim, S., Chu, T. L., Tsai, H. M., Ji, X., Zhang, J., Chee, E., \& Im, E. O. (2016). Practical issues in developing a culturally tailored physical activity promotion program for Chinese and Korean American midlife women: A pilot study. Journal of Medical Internet Research, 18(11), e303.

Chee, W., Kim, S., Tsai, H. M., \& Im, E. O. (2019). Decreasing sleep-related symptoms through increasing physical activity among Asian American midlife women. Menopause, 26(2), 152-161.

https://doi.org/10.1097/GME.0000000000001 178

Chee, W., Kim, S., Tsai, H. M., Liu, J., \& Im, E. O. (2020). Effect of an online physical activity promotion program and cardiovascular symptoms among Asian American women at midlife. Computers, Informatics, Nursing: CIN, 39(4), 198-207. https://doi.org/10.1097/CIN.00000000000006 69

Chesla, C. A., Chun, K. M., Kwong, Y., Gay, C. L., Chi, H.-L., Gu, Y., Hernandez, L., Huang, P., Strycker, L. A., \& Ma, J. (2016) Cultural adaptation of the group lifestyle balance program for Chinese Americans. The Diabetes Educator, 42(6), 686-696.

https://doi.org/10.1177/0145721716666679

Deng, F., Chen, D., Swartz, M. C., \& Sun, H. (2019).

A pilot study of a culturally tailored lifestyle intervention for Chinese American cancer survivors. Cancer Control, 26(1),
1073274819895489.

https://doi.org/10.1177/1073274819895489

Higgins, J. P. T., Thomas, J., Chandler, J., Cumpston, M., Li, T., Page, M. J., \& Welch, V. A. (2021). Cochrane Handbook for Systematic Reviews of Interventions. Retrieved April 26, 2021, from www.training.cochrane.org/handbook.

Im, E. O., Kim, S., Ji, X., Park, S., Chee, E., Chee, W., \& Tsai, H. M. (2017). Improving menopausal symptoms through promoting physical activity: A pilot web-based intervention study among Asian Americans. Menopause, 24(6), 653-662. https://doi.org/10.1097/GME.0000000000000 825

Jih, J., Le, G., Woo, K., Tsoh, J., Stewart, S., Gildengorin, G., Burke, A., Wong, C., Chan, E., Fung, L., Yu, F., Pasick, R., McPhee, S. J., \& Nguyen, T. T. (2016). Educational Interventions to Promote Healthy Nutrition and Physical Activity Among Older Chinese Americans: A Cluster-Randomized Trial. American Journal of Public Health (1971), 106(6), 1092-1098. https://doi.org/10.2105/AJPH.2016.303111

Keadle, S. K., McKinnon, R., Graubard, B. I., \& Troiano, R. P. (2016). Prevalence and trends in physical activity among older adults in the United States: A comparison across three national surveys. Preventive Medicine, 89, 3743.

https://doi.org/10.1016/j.ypmed.2016.05.009

Moher, D., Liberati, A., Tetzlaff, J., Altman, D. G., \& The PRISMA Group. (2010). Preferred reporting items for systematic reviews and meta-analyses: The PRISMA statement. International Journal of Surgery, 8, 336-341.

Page, M.J., McKenzie, J. E., Bossuyt, P. M., Boutron, I., Hoffmann, T.C., Mulrow, C.D., Shamseer, L., Tetzlaff, J. M., Akl, E. A., Brennan, S. E., Chou, R., Glanville, J., Grimshaw, J.M. , Hróbjartsson, A., Lalu, M. M., Li, T., Loder, E. W., Mayo-Wilson, E., McDonald, S., \& Moher, D. (2021). The PRISMA 2020 statement: an updated guideline for reporting 
systematic reviews. BMJ (Online), 372, n71n71. https://doi.org/10.1136/bmj.n71

Puciato, D., Borysiuk, Z., \& Rozpara, M. (2017). Quality of life and physical activity in an older working-age population. Clinical Interventions in Aging, 12, 1627-1634. https://doi.org/10.2147/CIA.S144045

Richardson, W. S., Wilson, M. C., Nishikawa, J., \& Hayward, R. S. (1995). The well-built clinical question: a key to evidence-based decisions. ACP Journal Club, 123(3), A12-13. https://doi.org/10.7326/ACPJC-1995-123-3-

A12
Sharma, M. (2017). Theoretical foundations of health education and health promotion (3rd ed.). Jones and Bartlett.

U.S. Department of Health and Human Services. (2018). Physical Activity Guidelines for Americans, 2nd edition. Washington, DC: U.S. Department of Health and Human Services. 
Appendix A: Figure 1. PRISMA flow diagram of literature search for physical activity interventions for older

\section{Identification}

Screening

Eligibility

Included

\section{Literature Search}

Keywords: Physical activity, sedentary behavior, intervention, older Asian American

Databases: MEDLINE, CINAHL, PubMed Central, and EBSCO databases

Limits: 1) Articles published in the past 5 years (2016-2021)

2) Peer-reviewed articles only

3) Articles published in English language only

4) Articles published in the United States only

5) Articles published on Asian Americans (55 years and older)

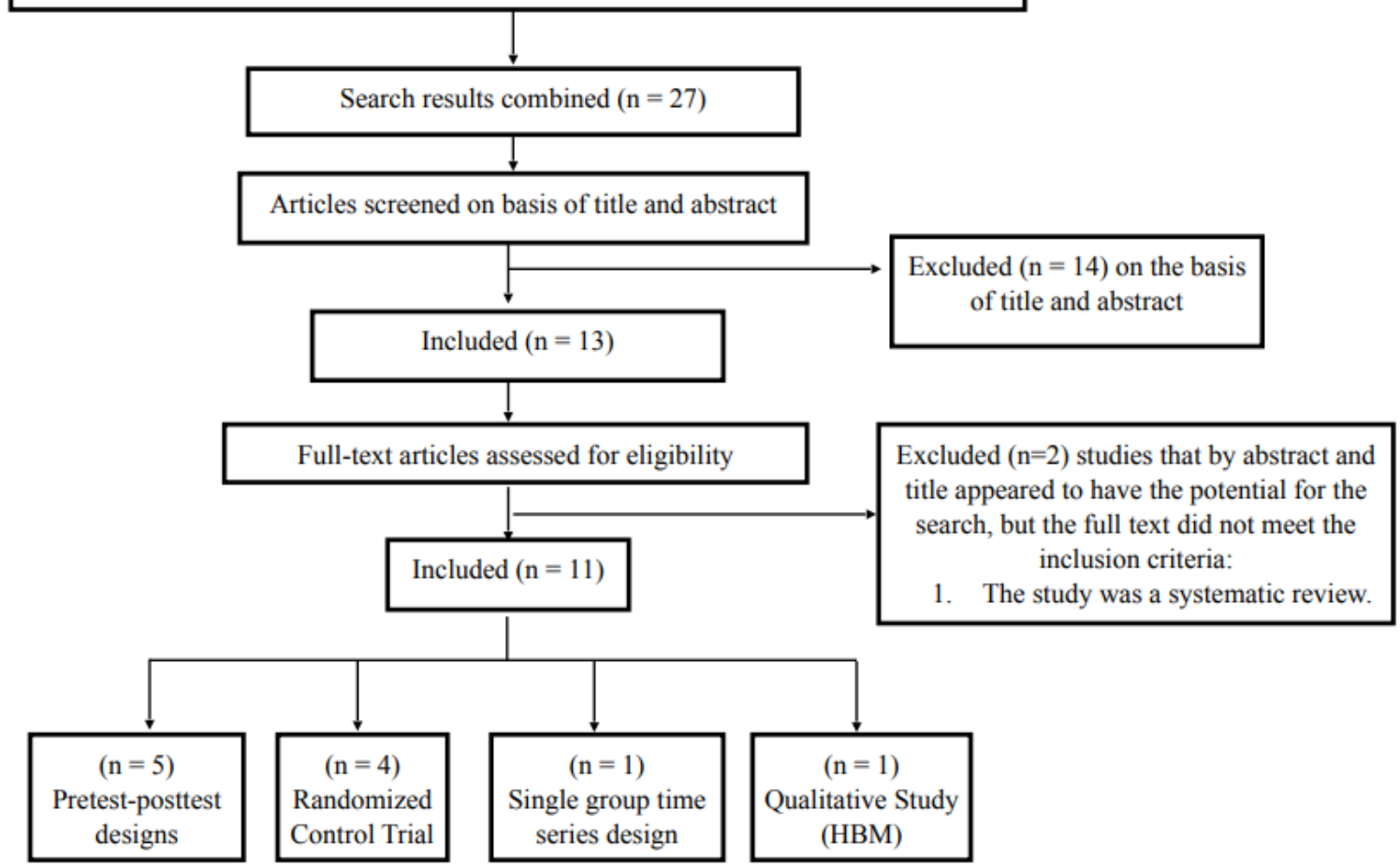


Appendix B: Table 1. PICO model as a literature search for inclusion criteria

\section{Explanation}

Population Older Asian American Adults (55+ years); based in the United States only

Intervention Any physical activity based program (educational, exercise, language-based, online, etc.)

\section{Comparison Absence of physical acitvity program}

Effectiveness of physical activity program; recommendation for

Outcome future physical activity intervention programs; beneficial health outcome of interests

Other

Published in a peer-reviewed academic journal in English. Articles

Published in the past 5 years (2016-2021) 
Appendix C: Table 2. Summary of physical activity (PA) interventions (2016-2021)

\begin{tabular}{|c|c|c|c|c|c|}
\hline $\begin{array}{l}\text { Study } \\
\text { No. }\end{array}$ & Author and Year & Type and Desreiption of Intervention & $\begin{array}{l}\text { Target Population } \\
\text { and Setting }\end{array}$ & $\begin{array}{l}\text { Study Design and } \\
\text { Sample Size }\end{array}$ & Outcome (effectiveness, post intervention, and retention) \\
\hline \#1 & Anet al, 2018 & $\begin{array}{l}\text { Practice-Based Intervention } \\
\text { Stroke Intervention program included in-person lectures and discussions } \\
\text { on PA and healthy eating choices. }\end{array}$ & $\begin{array}{l}\text { Korean American; } \\
\text { US }\end{array}$ & $\begin{array}{l}\text { Randomized Control Trials } \\
\mathrm{n}=73\end{array}$ & 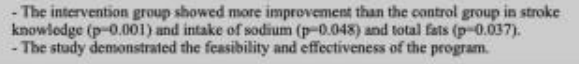 \\
\hline \#2 & Bender et al, 2017 & 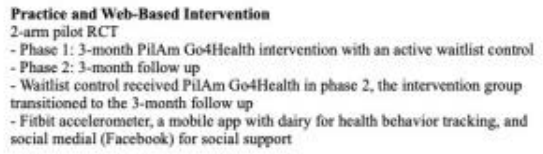 & $\begin{array}{l}\text { Filipino Americans; } \\
\text { Northem California }\end{array}$ & $\begin{array}{l}\text { Randomized Control Trials } \\
\mathrm{n}=45\end{array}$ & 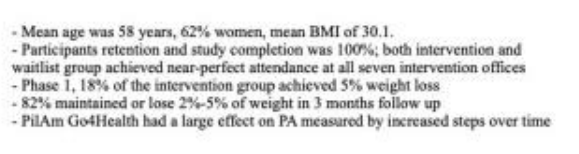 \\
\hline \#3 & Bender et al, 2018 & 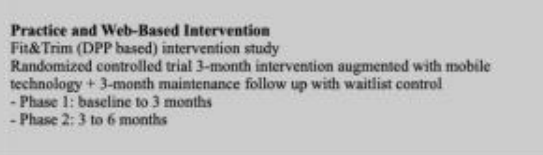 & Filipino Americans & $\begin{array}{l}\text { Randomized Control Trials } \\
n=67\end{array}$ & 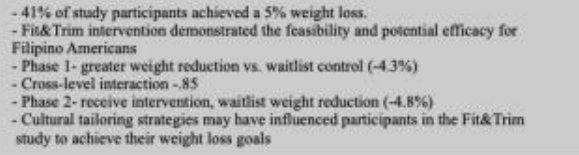 \\
\hline *4 & Chang et al, 2018 & 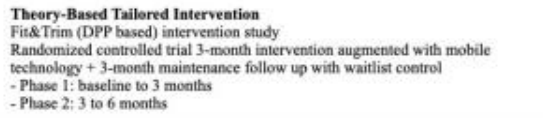 & $\begin{array}{l}\text { Chinese Americans } \\
\text { Korean Americans } \\
60 \text { years } \\
\text { Los Angeles, CA }\end{array}$ & $\begin{array}{l}\text { Qualitative Study design } \\
\mathrm{n}=132\end{array}$ & $\begin{array}{l}\text { Common themes by construct for stroke using a PA intervention: walling for exercise } \\
\text { - Soeme Chinese Amsericans focus groups believe stroke could be caused by vigorous } \\
\text { exercise, overeating, and extreme temperturec changes. } \\
\text { - Some Korean Americans stated risk factors for stroke included working too much } \\
\text { and exposure to cold temperatures. }\end{array}$ \\
\hline \#5 & Chee et al, 2016 & $\begin{array}{l}\text { Language and Web-Based Tailored Intervention } \\
\text { Phase l: usability test and expert review } \\
\text {-5 Asian American midlife women participated for both usability and expert } \\
\text { review } \\
\text { Phase 2: a preliminary randomized trial } \\
\text { - Pretest, post } 1 \text { moath (time point 1), post } 3 \text { months (time point 2) }\end{array}$ & $\begin{array}{l}\text { Chinese Americans } \\
\text { Korean Amerieans } \\
\text { Midlife Wamen }\end{array}$ & $\begin{array}{l}\text { Pretest - Posttest desizns } \\
\mathrm{n}=69\end{array}$ & 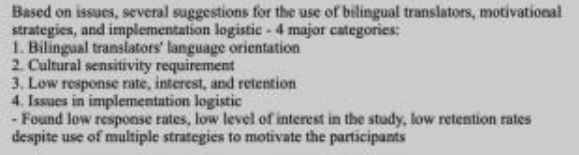 \\
\hline \#6 & Chee et al, 2019 & $\begin{array}{l}\text { Practice and Web-Based Intervention } \\
\text { Mesured background characteristics, sleep-related symptoms, PA experiences } \\
\text { at pretest, post } 1 \text { month, post } 3 \text { month }\end{array}$ & $\begin{array}{l}\text { Asian American } \\
\text { midlife women }\end{array}$ & $\begin{array}{l}\text { Pretest - Posttest designs } \\
\mathrm{n}=26\end{array}$ & 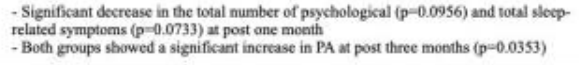 \\
\hline *7 & Chee et al, 2020 & 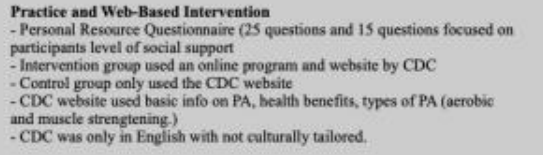 & $\begin{array}{l}\text { Asian American } \\
\text { midlife women ; } \mathrm{CA}\end{array}$ & $\begin{array}{l}\text { Pretest - Posttest designs } \\
n=26\end{array}$ & 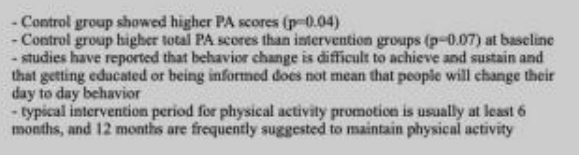 \\
\hline \# & Chesha ef al, 2016 & $\begin{array}{l}\text { Language and Practice-Based Tailered Intervention } \\
16 \text { session program was administered over six months in separate Mandarin } \\
\text { (n-9) and English (n-16) } \\
\text { - Assessed baseline, three months, six months }\end{array}$ & Chinese Americans & $\begin{array}{l}\text { Single-Group Time Series } \\
\text { Design } \\
\mathrm{n}=25\end{array}$ & 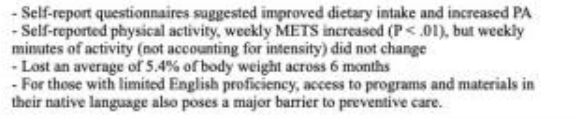 \\
\hline H9 & Deng et al, 2019 & $\begin{array}{l}\text { Language and Practice-Based Tailored Intervention (Community-Based) } \\
\text {-RENEW-C (The Reach out to ENhunoeE Wellness program workbook } \\
\text { transilated into Mandarin Chinese with additional PA and dietary information } \\
\text {-50-week program }\end{array}$ & $\begin{array}{l}\text { Chinese Americuns, } \\
\text { Houston, TX }\end{array}$ & $\begin{array}{l}\text { Pretest - Posttest designs } \\
n=55\end{array}$ & $\begin{array}{l}\text { - Participants reported significantly higher consumption of vegetables and higher } \\
\text { frequerny of PA at post-intervention sarney } \\
\text { - Postintervention } 96 \% \text { of the participants walked at least } 30 \text { minutes every day } \\
\text { compared to } 86 \% \text { at baseline }\end{array}$ \\
\hline \#10 & Im et al, 2017 & $\begin{array}{l}\text { Practice and Web-Based latervention } \\
\text { 3-month pilot randomized Web-based PA promotion programb with educational } \\
\text { modules on menopausal symptoms. } \\
\text { - Midlife Women's Symptome Index } \\
\text { - Kaiser Physical Activity Sarvey }\end{array}$ & $\begin{array}{l}\text { Asian American } \\
\text { miflife women }\end{array}$ & $\begin{array}{l}\text { Pretest - Posttest designs } \\
n=29\end{array}$ & 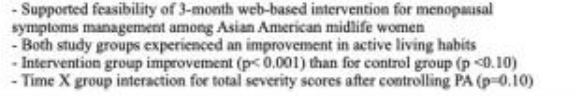 \\
\hline$\$ 11$ & Jih et al, 2016 & 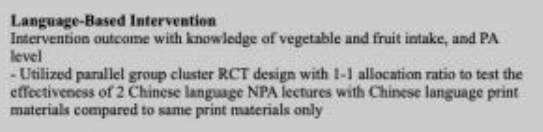 & $\begin{array}{l}\text { Chinese American; } \\
\text { ages } 50 \text { to } 75 \text {. } \\
\text { San Franciso, } \mathrm{CA}\end{array}$ & $\begin{array}{l}\text { Randomized Control Trials } \\
\mathrm{n}=756\end{array}$ & 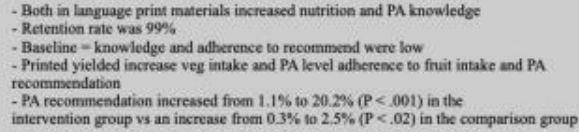 \\
\hline
\end{tabular}

